\title{
INCORPORAÇÃO DE MODELAGEM DA INFORMAÇÃO EM PROTOTIPAGEM DIGITAL ${ }^{1}$
}

\author{
DIGITAL FABRICATION INCORPORATING BUILDING INFORMATION \\ MODELING
}

\author{
Victor Calixto \\ Universidade Estadual de Campinas (UNICAMP) \\ calixto@fec.unicamp.br \\ Euler José de Oliveira Morais \\ Universidade Estadual de Campinas (UNICAMP) \\ contato@eulermorais.com.br \\ Regina Coeli Ruschel \\ Universidade Estadual de Campinas (UNICAMP) \\ ruschel@fec.unicamp.br
}

\section{Resumo}

Com o avanço da modelagem tridimensional as formas livres que eram difíceis de serem representadas e calculadas pelas ferramentas tradicionais de projeto puderam ser representadas e fabricadas através das máquinas de controle numérico. A prototipagem digital surge nesse cenário com um novo sistema de produção de modelos em escala. A Modelagem da Informação da Construção (BIM) surge como uma nova abordagem no desenvolvimento dos processos da prototipagem digital. Os objetos gerados já não são meras representações geométricas, mas agregam propriedades especificas como: tipo de material, dimensões parametrizadas, custos, entre outras. Nesse artigo será desenvolvida uma demonstração conceitual de como o BIM pode ser incorporado aos sistemas de produção automatizada de modelos em escala no âmbito da preparação da informação do protótipo. Tomou-se como base um estudo de caso de produção digital realizado em CAD. Um balcão projetado para o Museu Exploratório de Ciências da UNICAMP idealizado por Wilson Barbosa Neto em sua dissertação em 2013 na UNICAMP. Primeiramente foi realizado um levantamento da preparação da informação no protótipo. Em seguida o processo foi remodelado em BPMN (Business Process Model and Notation). Utilizando-se do Guia BIM (Building Information Modeling Project Execution Planning Guide), o estudo de caso foi remodelado em BPMN-BI a partir do qual foram extraídas informações as quais foram analisadas. Conclusões foram discutidas a partir da observação dos dados.

Palavras-chave: Prototipagem digital. BIM. CAD.

\section{Abstract}

With the advancement of three-dimensional modeling the free forms that were difficult to be represented and calculated by the traditional tools of design could be represented and constructed through computer numerical control. The digital prototyping arises in this scenario as a new scaled models production system. The Building Information Modeling (Building Information Modeling - BIM) emerges as a new approach in the development of digital prototyping processes. The objects generated are no longer mere geometric representations but add specific properties such as material type, parameterized dimensions, and costs, among others. In this paper will be

${ }^{1}$ CALIXTO, Victor; MORAIS, Euler José de Oliveira; RUSCHEL, Regina Coeli. TIC2015. In: ENCONTRO BRASILEIRO DE TECNOLOGIA DE INFORMAÇÃO E COMUNICAÇÃO NA CONSTRUÇÃO, 7., 2015, Recife. Anais... Porto Alegre: ANTAC, 2015. 
developed a conceptual demonstration of how BIM can be incorporated into automated production systems of scaled models under the prototype information preparation. Was taken based on a study of digital prototyping held in CAD. A desk table designed for Exploratory Museum of UNICAMP Sciences. It was designed by Wilson Barbosa Neto in his dissertation in 2013 at UNICAMP. First was made a survey of information preparation in the prototype. Then the process was refurbished in BPMN (Business Process Model and Notation). Using BIM Guide the case study was again renovated in BPMN-BIM. From the BPMNs refurbished data were extracted and analyzed. Conclusions were discussed from the observation data.

Keywords: Digital Prototyping. BIM. CAD.

\section{INTRODUÇÃO}

O avanço das tecnologias e ferramentas de CAD (Computer Aided Design) juntamente com o desenvolvimento da potência de cálculo dos computadores abre uma série de possibilidades aos arquitetos para explorarem o desenvolvimento de formas complexas em projeto. Segundo Moore (1956 apud KURZWEIL 2005) a capacidade de processamento dos computadores dobra a cada dois anos. Esse aumento de capacidade tem influenciado todos os campos do conhecimento inclusive o pensar e o fazer arquitetônicos.

Como consequência os métodos de modelagem tridimensional começaram a ser largamente utilizados. Eles oferecem aos projetistas novas ferramentas como: (i) modelagem paramétrica, (ii) linguagens scripts embutidas, (iii) visualização arquitetônica em vários níveis de representação (wireframe, solid, $x$-ray, entre outras), (iv) imagens foto realísticas. De acordo com Kolarevic (2003, p.118), o avanço da modelagem 3D tem sido impulsionado com a utilização de curvas Non Uniform Rational Basis Spline (NURBS). As formas livres que eram difíceis de serem representadas e calculadas pelas ferramentas de projeto tradicionais tornaram-se acessíveis com a introdução das NURBS. Começaram a serem fabricadas de forma automatizada através de máquinas controladas numericamente, fabricação CAM (Computer Aided- Manufacture). Segundo Puppo (2009, p.5) a ligação entre os processos de concepção e construção é mais direta, pois a informação é rapidamente extraída, usada e compartilhada com mais facilidade e velocidade.

A prototipagem digital surge nesse cenário com um novo sistema de produção de modelos em escala. São utilizadas matérias primas como gesso, resina, plásticos, madeira. Os modelos são executados em prazos mínimos se comparados às técnicas tradicionais (PUPO, 2009 , p. 2) e têm sido desenvolvidos tradicionalmente dentro do sistema CAD. A Modelagem da Informação da Construção (Building Information Modeling - BIM) surge como uma nova abordagem no desenvolvimento dos processos da prototipagem digital. Os objetos gerados já não são meras representações geométricas mas agregam propriedades especificas como: tipo de material, dimensões parametrizadas, custos, entre outras.

Nesse artigo será desenvolvida uma demonstração conceitual de como o BIM pode ser incorporado aos sistemas de produção automatizada de modelos em escala no âmbito da preparação do protótipo. Na Fundamentação definiu-se o conceito de modelo, suas tipologias e os cinco passos para sua a produção. Introduziu-se o conceito de BIM em itens relevantes como: (i) interoperabilidade, (ii) modelos orientados a objetos, (iii) avaliação, quantitativos e custos. No Método tomou-se como base um estudo de caso de produção digital realizado em $C A D$. Foi feito um levantamento da preparação da informação no protótipo. Esse estudo foi remodelo em BPMN. Utilizando-se do Guia BIM (COMPUTER, 2011) o estudo de caso foi remodelado de modo a atender os seus itens seguindo o padrão $B I M$. Essa remodelagem foi refeita em BPMN. No item Resultados e Discussão foram analisados e comparados os BPMNs $(C A D$ e $B I M)$.

\section{FUNDAMENTAÇÃO}

De acordo com Celani e Piccoli (2010 p. 52) existem cinco passos para a produção de um 


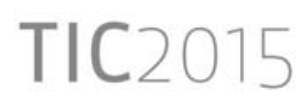

modelo em escala através da prototipagem digital. No primeiro passo define-se o objetivo do modelo, escala e configuração. No segundo passo são definidas as técnicas de produção e os materiais a serem utilizados. Prepara-se os arquivos de produção do modelo no terceiro passo. No quarto passo é feito a produção do modelo através de maquinas como FDM (Fused Deposition Modeling), SLS (Selective Laser Synthering), 3DP (Inkjet 3D printing), SLA (Stereolithography), Polyjet,Lom, Laser Cutter, Vinyl Cuter, CNC Router, entre outras. No quinto e último passo, dependendo da técnica empregada, monta-se o modelo ou dá-se acabamento ao objeto. A Figura 1 demonstra o modelo proposto dentro do sistema CAD.

Figura 1 - As cinco etapas da produção de um modelo em escala com as técnicas de prototipagem rápida

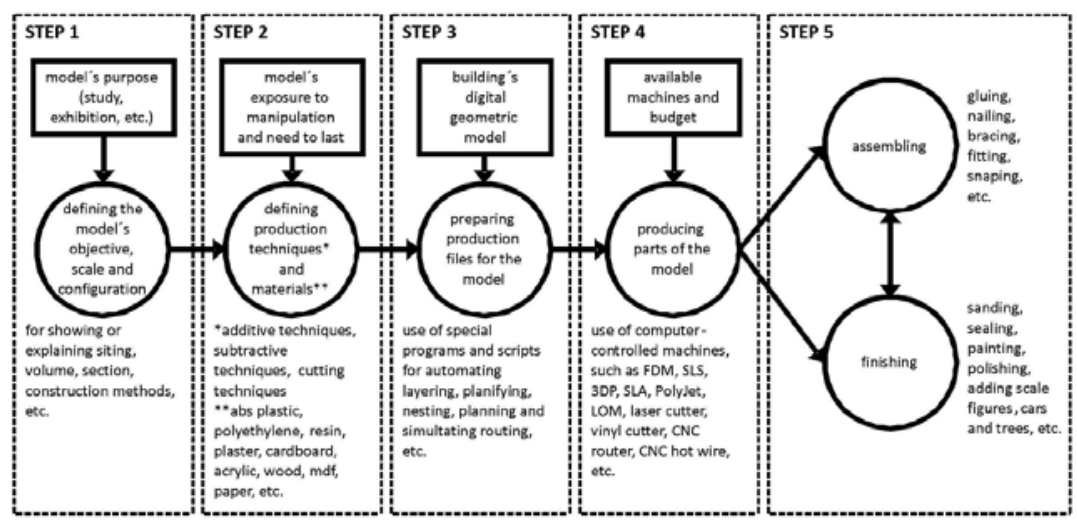

Fonte: Celani e Piccoli (2009, p. 52)

Nesse caso o modelo gerado é representado apenas geometricamente sem propriedades agregadas como ocorre em modelos gerados em BIM. Esse sistema ao agregar informações ao objeto possibilita uma nova abordagem com relação a preparação da informação nos protótipos.

\section{MÉTODO}

Foi desenvolvido para demonstrar conceitualmente como o BIM pode ser incorporado ao processo de prototipagem digital. A partir de um estudo de caso em sistema CAD fez-se o levantamento de informações do processo de preparação da informação no protótipo, construindo um modelo teórico em linguagem BPMN. O segundo passo foi criar um mesmo modelo BPMN do processo de preparação da informação de protótipos dentro do sistema BIM tomando como referência o Guia BIM (COMPUTER, 2011). O terceiro passo foi analisar os dois modelos discutindo recursos associados a modelagem da informação como: (i) a interoperabilidade, (ii) a modelagem orientada a objetos, (iii) avaliações do modelo, extrações de quantitativos e suporte a estimativa de custos, (iv) a extração de vistas.

\section{RESULTADOS E DISCUSSÃO}

\subsection{Levantamento da preparação da informação no protótipo em CAD}

O estudo de caso escolhido para esta pesquisa foi um balcão projetado para o Museu Exploratório de Ciências da UNICAMP. Foi idealizado por Wilson Barbosa Neto em sua 
dissertação no ano de 2013 na UNICAMP. Segundo Barbosa (2013, p. 68), o conceito do balcão deveria abarcar a missão do Museu de promover a disseminação da cultura e inovação tecnológica, por isso, deveria ser inovador, interessante, e, ao mesmo tempo, leve e fácil de mover.

A pesquisa de Barbosa (2013) teve como propósito documentar de maneira sistemática o processo e as etapas da fabricação digital desde sua concepção até o processo de fabricação na indústria. $O$ trabalho se desenvolveu através de uma pesquisa ação sendo dividido em 3 etapas: (i) descrição do projeto, (ii) estágio exploratório e (iii) estagio de fabricação.

O estágio abordado nesse artigo será o exploratório. Segundo Barbosa (2013, p. 67), o estágio exploratório envolveu atividades de modelagem e análise virtual; prototipagem rápida e fabricação digital em escala laboratorial, assim como exercícios de investigação de materiais, potencialidades e limitações dos equipamentos CNC (Computer Numerical Control) na escala industrial.

O protótipo foi primeiramente redesenhado de acordo com o modelo de processo de fabricação digital proposto por Celani e Piccoli (2010, p. 52). O objetivo do primeiro protótipo produzido foi "analisar a estabilidade das partes e as possíveis combinações de layout" Barbosa (2013, p. 78). O método empregado foi contouring, sendo construído em gesso numa máquina de PP (Plaster Based 3d Printer) também conhecida como 3DP. A Figura 2 mostra o registro de todo o processo até o modelo final.

Figura 2 - Processo de modelagem para análise de estabilidade e layout

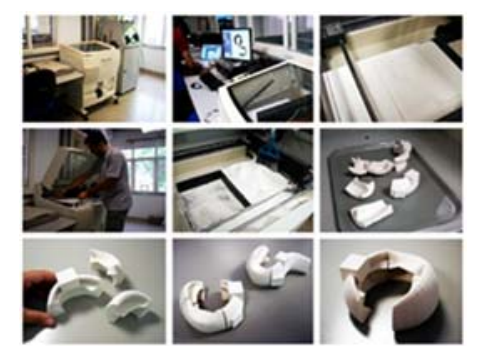

Fonte: Barbosa (2013, p. 84)

A escala escolhida para o modelo foi a 1:10. Foi necessário o desenvolvimento de um modelo sólido que fosse exportado em stl para que pudesse ser interpretado pela máquina Z-corp. A Figura 03 ilustra esse processo.

Figura 3 - Primeiro protótipo redesenhado conforme modelo de fabricação digital

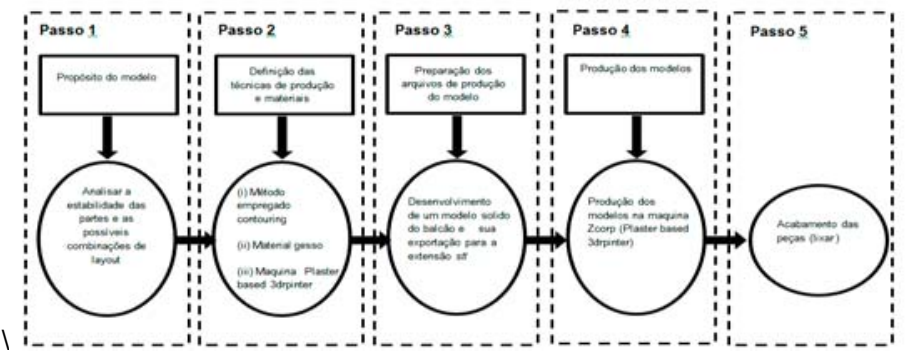

Fonte: Os autores e adaptado de Celani e Piccoli (2010, p. 52) 


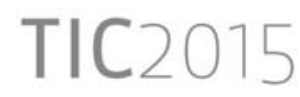

Esses dados alimentam uma segunda etapa, a produção do modelo através de um material laminado. O objetivo desse modelo foi identificar possíveis problemas de encaixe no processo industrial. O método de fabricação digital empregado foi sectioning, a máquina a Laser Cutter e o material utilizado foi papel paraná de $1 \mathrm{~mm}$. A Figura 4 ilustra o processo.

Figura 4 - Processo de modelagem para análise de encaixes

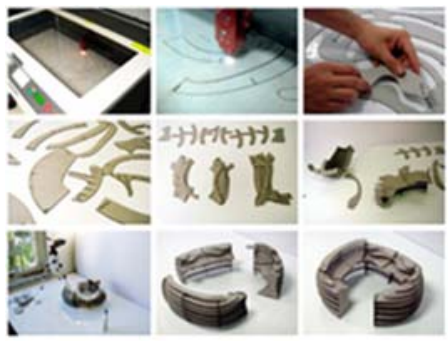

Fonte: Barbosa (2013, p. 84)

A preparação dos arquivos teve como primeiro passo seccionar o modelo em planos horizontais e verticais, a fim de extrair as prateleiras do modelo e secciona-lo em três partes que compõem o balcão. O protótipo foi desenvolvido com encaixes do tipo "grelha" com o intuito de travar as peças perpendicularmente e sendo organizadas para corte em impressora 3D em plano horizontal e dentro das dimensões do material que foram confeccionadas. Após esse processo, o arquivo é salvo pelo programa AutoCAD na extensão $d w g$, que o formato de arquivo suportado pela máquina de Laser Cutter. Nas propriedades de plotagem são ajustadas as configurações de velocidade e potência da máquina de acordo com o tipo de material, nesse caso papel paraná. As dimensões das chapas de papel paraná utilizadas respeitam as dimensões da máquina que eram de $450 \times 800 \mathrm{~mm}$ e espessura do material era de $1 \mathrm{~mm}$. A última etapa é o processo de montagem e colagem das peças. A Figura 4 ilustra as etapas.

Figura 4 - Segundo protótipo redesenhado conforme modelo de fabricação digital

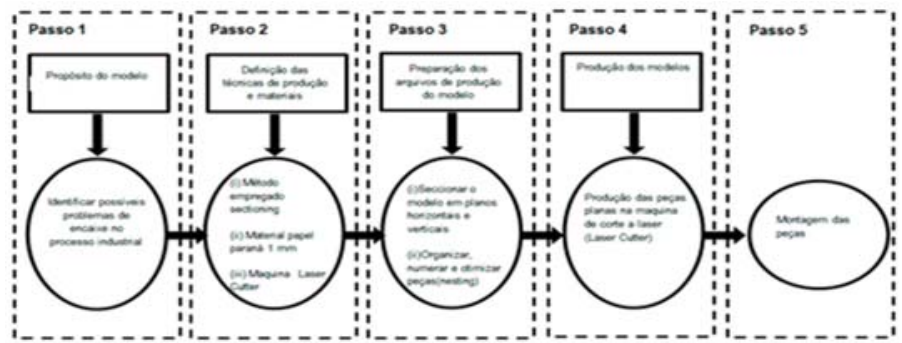

Fonte: os autores, adaptado de Celani e Piccoli (2010, p.52)

Os procedimentos acima são complementares e fazem parte de um processo completo. Para cada fase verifica-se se o modelo atende ao propósito. Caso isso não ocorra, volta-se novamente ao ciclo na fase de preparação dos arquivos de produção do modelo. Se atende a todos os procedimentos então passa-se adiante para o desenvolvimento do modelo para fabricação digital. O processo completo pode ser observado na Figura 5. 
Figura 5 - Processo completo estudo de caso de fabricação digital

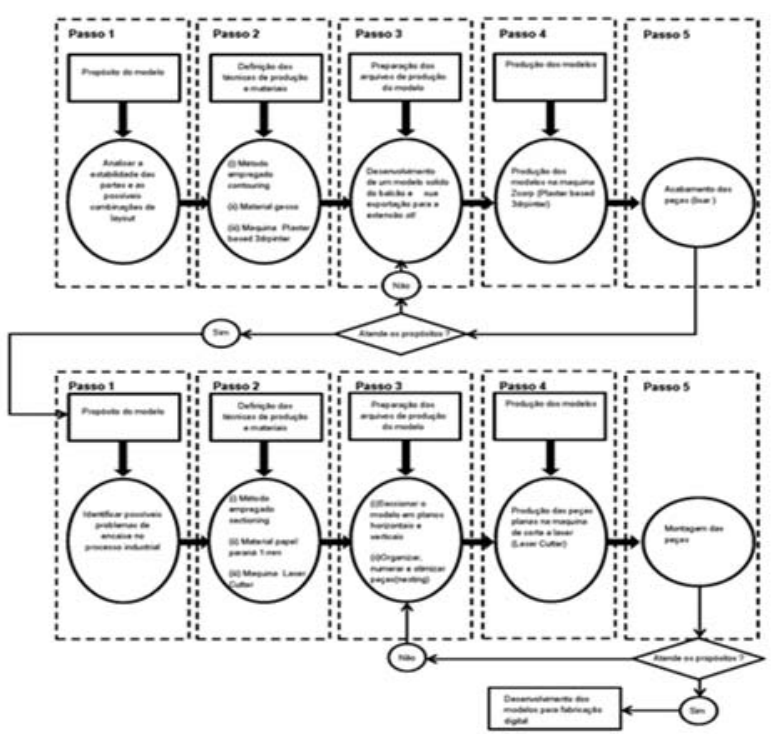

Fonte: os autores, adaptado de Celani e Piccoli (2010, p. 52)

\subsection{Remodelagem CAD em BPMN}

A partir do modelo completo é possível reconstruir o processo através da Notação de Modelagem de Processos de Negócio (Business Process Model and Notation - BPMN). BPMN é uma notação da metodologia de gerenciamento de processos de negócio e trata-se de uma série de ícones padrões para o desenho de processos, que facilita o entendimento do usuário.

O processo segue com os seguintes passos: (a) concepção paramétrica do modelo que gera um arquivo de Grasshopper de extensão gh ; (b) definição do propósito do modelo; (c) definição das técnicas e materiais; (d) preparação dos arquivos de produção do modelo em Rhinoceros; (e) envio para máquina Zcorp; (f) acabamento da peça (lixar) ; (g) verificação do atendimento aos propósitos: caso atendida segue-se ao passo $\mathrm{h}$, caso contrário volte-se ao passo d; (h) definição do propósito do novo modelo; (i) definição das técnicas de produção e materiais do novo modelo; (j) ajuste paramétrico do modelo em Rhinoceros e Grasshopper (k) exportação das vistas $2 \mathrm{~d}$ em Rhinoceros para $d w g$; (l) preparação dos arquivos de produção do modelo em AutoCAD; $(\mathrm{m})$ envio para máquina de corte a laser; $(\mathrm{n})$ montagem da peça; (o) verificação de atendimento dos propósitos do novo modelo: atendendo segue-se para passo $p$, caso contrário volta-se ao passo k; $(p)$ desenvolvimento dos modelos para fabricação digital; (q) fim do processo. Na Figura 6 pode-se observar todo o desenvolvimento. 
Figura 6 - Remodelagem CAD em BPMN

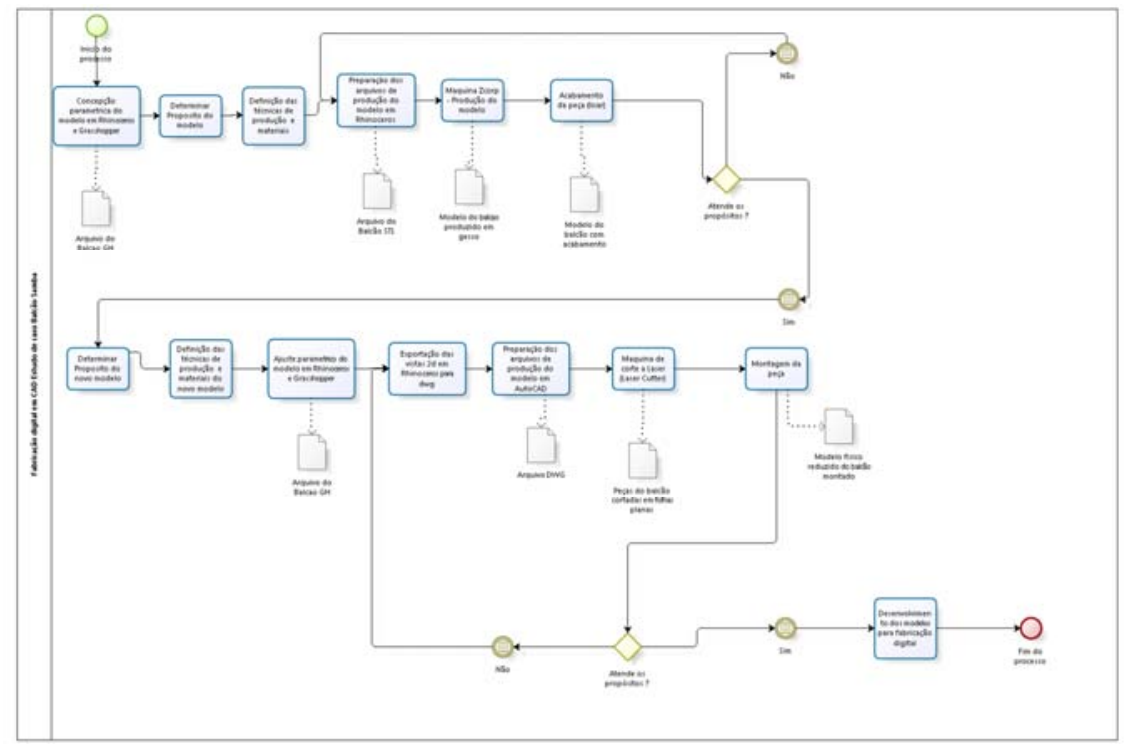

Fonte: os autores

\subsection{Remodelagem BIM em BPMN (Guia BIM)}

O ambiente de modelagem BIM escolhido para a remodelagem do processo em BPMN foi o Revit. É um ambiente amplamente difundido através de toda a plataforma de softwares BIM desenvolvida pela a empresa AutoDesk conforme pesquisas em vários países. Segundo Khosrowshahi e Arayici (2012, p.623) é o software mais conhecido no Reino Unido. O processo da remodelagem BIM em BPMN segue os seguintes passos: (a) concepção paramétrica do modelo em Revit que gera um arquivo rvt; (b) definição do propósito do modelo; (c) definição das técnicas de produção e materiais; (d) exportação do modelo rvt para o formato sat; (e) preparação dos arquivos de produção do modelo em AutoCAD (arquivo stl); (f) envio para maquina Zcorp; (g) acabamento da peça (lixar); (h) verificação de atendimento dos propósitos com duas alternativas de continuidade do processo: caso atenda aos propósitos dirige-se ao passo i , caso contrário volta-se ao passo d; (i) propósito do novo modelo; (j) definição das técnicas de produção e materiais do novo modelo; (k) ajuste paramétrico do modelo em Revit (gera um arquivo rvt); (I) exportação das vistas 2D do formato rvt para o formato $d w g ;(\mathrm{m})$ preparação dos arquivos de produção do modelo em AutoCAD (n) envio para máquina de corte a laser; (o) montagem da peça; ( $p$ ) verificação de atendimento aos propósitos do novo modelo, atendendo dirige-se ao passo $q$, caso contrário volta-se ao passo l; (q) desenvolvimento dos modelos para fabricação digital; (r) fim do processo. A Figura 08 demonstra todo o desenvolvimento. 


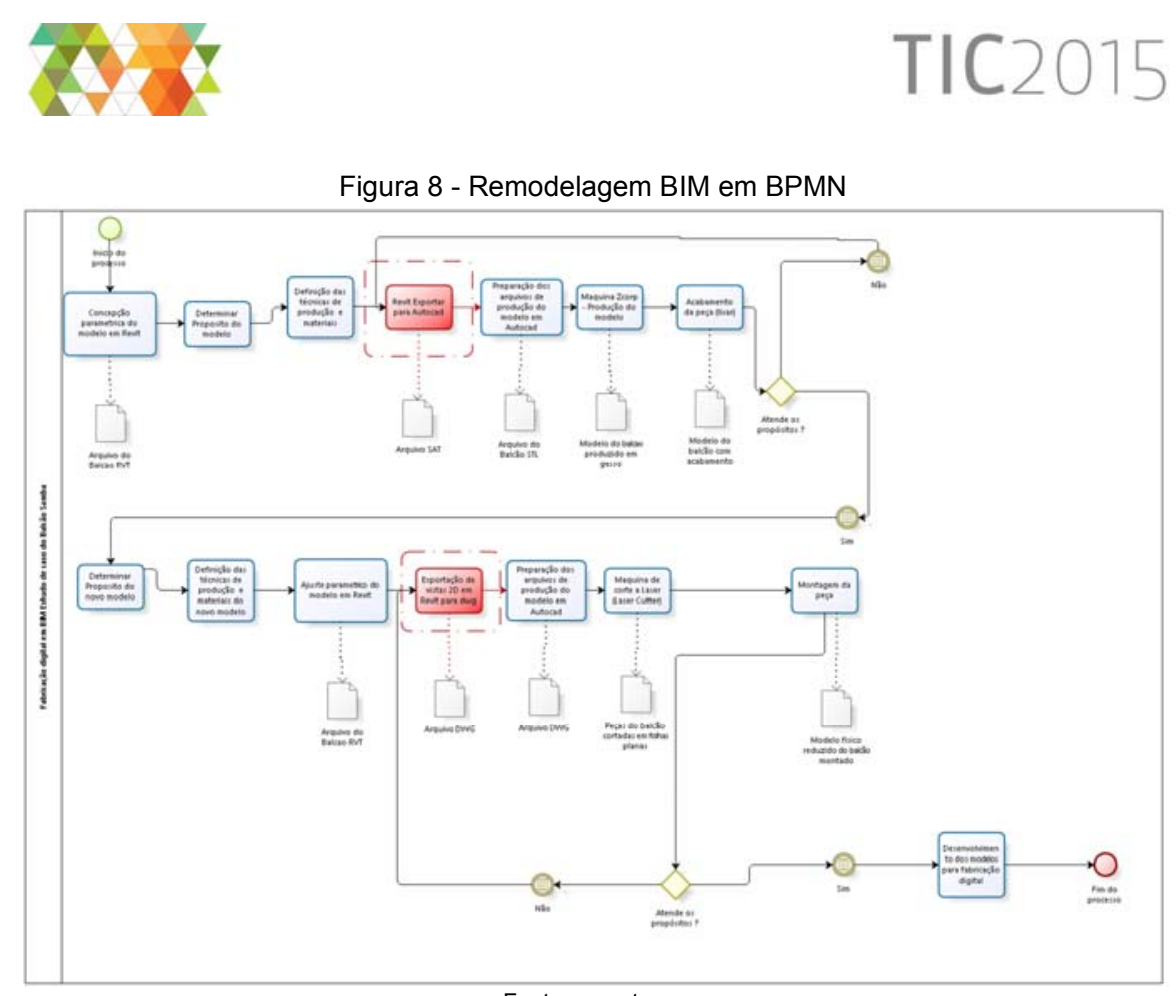

Fonte: os autores

Ao se verificar o processo, percebe-se que há uma quebra no processo de transferência da informação. $\mathrm{Na}$ etapa de preparação dos arquivos de produção do modelo verifica-se a necessita de uma extensão reconhecida pelas máquinas de fabricação. É nessa fase que as informações e propriedades contidas no modelo BIM se perdem. Os softwares BIM não exportam o modelo no formato utilizado pelas máquinas de prototipagem digital. As informações são sempre relativas ao objeto real e não referentes a um modelo ou protótipo. Fica clara a necessidade de um elemento que faça a conexão necessária evitando a quebra no fluxo da informação, que possibilite o modelo seja reutilizado. Existe um plug-in que torna possível a exportação dos arquivos do Revit diretamente para a extensão stl. Este plug-in pode ser encontrado no site https://apps.exchange.autodesk.com/en. Neste site existem plugins de interoperabilidade para todos os produtos AutoDesk, o que torna possível a remodelagem do processo em BPMN, de maneira otimizada conforme ilustra a Figura 9.

O processo segue os passos para a remodelagem BIM em BPMN, otimizada : (a) concepção paramétrica do modelo em Revit que gera um arquivo rvt ; (b) definição do propósito do modelo; (c) definição das técnicas de produção e materiais; (d) preparação dos arquivos de produção do modelo no plug-in de exportação em Revit (arquivo rvt para stl); (e) envio para maquina Zcorp; (f) acabamento da peça (lixar); (g) verificação de atendimento aos propósitos: caso atenda segue-se para o passo h, caso contrário volta-se ao passo d; (h) propósito do novo modelo; (i) definição das técnicas de produção e materiais do novo modelo; (j) ajuste paramétrico do modelo em Revit (gera um arquivo rvt); (k) exportação das vistas $2 \mathrm{~d}$ em rvt para dwg; (l) preparação dos arquivos de produção do modelo em AutoCad; $(\mathrm{m})$ envio para máquina de corte a laser; (n) montagem da peça; (o) verificação do atendimento dos propósitos do novo modelo, atendendo segue-se para passo q, caso contrário volta-se ao 


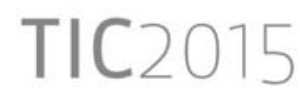

passo I; (p) desenvolvimento dos modelos para fabricação digital; (q) fim do processo. A Figura 9 demonstra todo o desenvolvimento.

Figura 9 - Remodelagem BIM em BPMN, otimizada[rrr1]

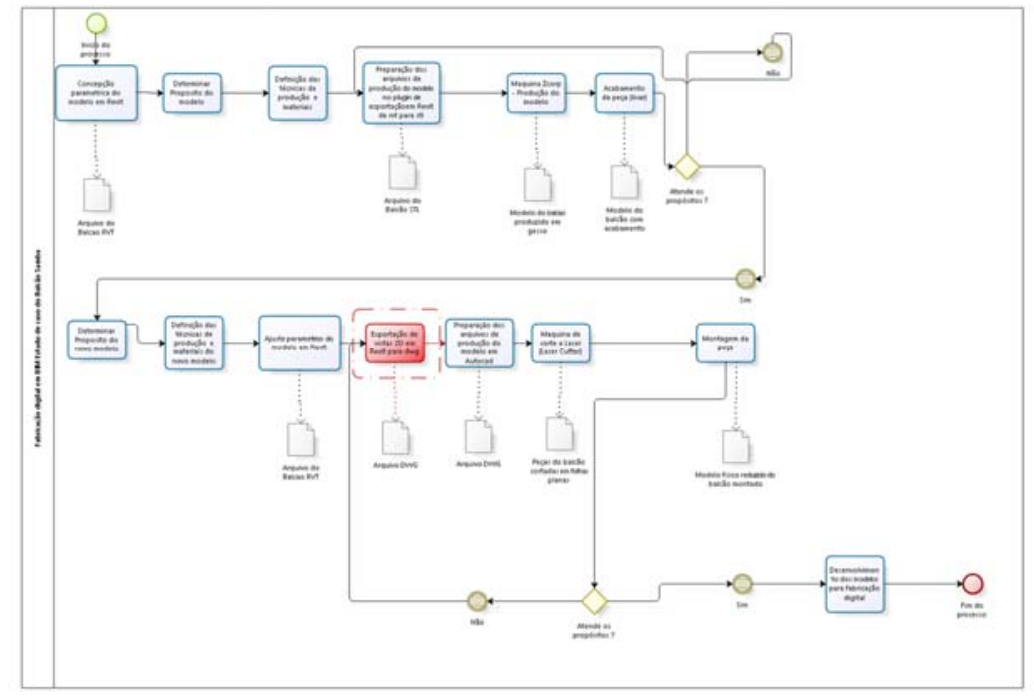

Fonte: os autores

\subsection{Análise e discussões}

Através dos gráficos BPMN foi possível identificar informações importantes sobre o processo de prototipagem digital. Estabeleceu-se comparações, concluindo sobre o impacto que o sistema BIM pode ter sobre esse processo. Um avanço na prototipagem digital seria que os arquivos para produção pudessem trazer incorporadas informações sobre o modelo num fluxo contínuo de informações. O modelo hipotético descrito na Figura 10 demonstra as características paramétricas envolvidas na criação de um protótipo e que deveriam estar incorporados a ele.

Figura 10 - Modelo hipotético

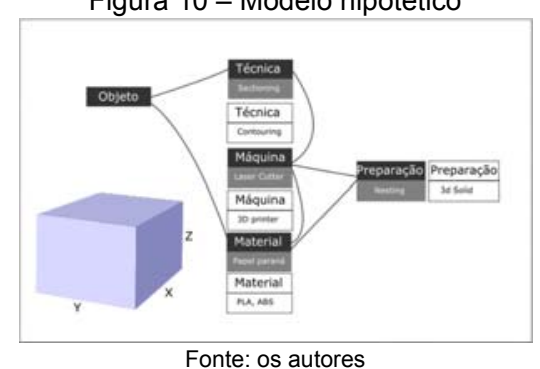

Fonte: os autores 
Pode-se observar primeiramente a parametrização da geometria do objeto representado por $x, y$ z. As informações relativas ao objeto estão inseridas, porém nem todas são utilizadas. Pode-se verificar que a técnica e o material utilizado estão diretamente relacionados ao modelo. No caso a técnica é o sectioning e o material o material é o papel paraná. A técnica está relacionada com a máquina, ou seja, dependendo da técnica escolhida existe o maquinário adequado à execução. A máquina por sua vez está relacionada ao material. Diferentes maquinários trabalham com materiais específicos. No caso a máquina adequada ao material papel paraná é a Laser Cutter. A preparação da informação foi feita por nesting e está relacionada com o material e a máquina. Todas essas informações se relacionam simultaneamente no protótipo de maneira parametrizada. Modelos como estes tornam mais eficiente o processo de fabricação digital em BIM, porém existem desafios a serem superados.

O primeiro desafio é que no panorama atual nenhum software agrega informações ao protótipo como no modelo hipotético idealizado. Ocorre que as extensões liberadas pelos softwares não agregam informações paramétricas o que acaba interrompendo o fluxo das informações durante o processo.

O segundo desafio está relacionado com o fluxo de informações entre as máquinas de prototipagem digital e os softwares BIM.

Com base em todas as informações analisadas, na avaliação do modelo hipotético e nos desafios que se apresentaram podem-se extrair algumas conclusões. Fica evidente que a troca de informações é deficiente e existe uma demanda de desenvolvimento nesse setor. A AutoDesk desenvolveu um plug-in para estabelecer a interoperabilidade entre o Revit e máquinas aditivas de prototipagem digital. Com isso é possível transformar arquivos rvt em formato stl. O que se observa é que esse processo em nada difere do desenvolvido em CAD. Todas as informações que poderiam ser agregadas ao modelo em escala não são inseridas.

O Revit não dá o suporte necessário para que a transferência de informações para esses modelos ocorra. Isso é uma ruptura do processo. Ao se analisar essa situação a luz das possíveis relações paramétricas entre as propriedades materiais e as técnicas de produção em fabricação e prototipagem digital identifica-se quatro caminhos para o desenvolvimento de soluções desse processo:

- desenvolvimento de plug-ins (que atendam a demanda de informação necessária para um modelo de prototipagem digital);

- desenvolvimento de softwares específicos dentro de uma plataforma (que atendam a demanda de informação necessária para um modelo de prototipagem digital);

- desenvolvimento de softwares independentes com interoperabilidade, leitura e escrita em ifc e exportação para formatos específicos das maquinas de prototipagem (que atendam a demanda de informação necessária para um modelo de prototipagem digital);

- agregar informações (que atendam a demanda de informação necessária para um modelo de prototipagem digital) e adicionar a leitura das extensões BIM em máquinas de prototipagem digital.

A demanda de informações necessárias a um modelo de prototipagem digital que são:

- definições paramétricas das dimensões do modelo (transformações e escala);

- definições paramétricas de materiais (propriedades e dimensões);

- definições paramétricas da técnica de produção (Impressão 3d, Corte a Laser, CNC);

- definições paramétricas das maquinas de produção;

- definições paramétricas da produção;

- simulação da produção e montagem; 
- quantitativos e custos dos materiais e produção (quantidade de material, taxa de desperdício, custo do tempo de produção).

Então surge a necessidade da remodelagem do estudo de caso utilizando-se BPMN - BIM. Para tanto foi escolhido o caminho de desenvolvimento de plug-ins tendo como fatores de orientação as demandas de informações necessárias ao modelo de prototipagem digital. O objetivo é verificar quais impactos podem ser observados ao se incorporar na produção de modelos em escala o sistema BIM utilizando-se do software de modelagem Revit.

O processo segue os seguintes passos: (a) concepção paramétrica do modelo em Revit; (b) propósito do modelo; (c) plug-in de fabricação digital e prototipagem rápida; (d) \{a\} Máquina Zcorp \{b\} Máquina de corte a laser; (e) \{a\} Acabamento da peça \{b\} Montagem da peça; (f) Verificação de atendimento aos propósitos do modelo: caso atendido segue-se para o passo g, caso contrário volta-se ao passo c; (g) fim do processo de prototipagem digital. A Figura 11 demonstra todo o processo.

Figura 11 - BPMN-BIM utilizando-se plug-in hipotético

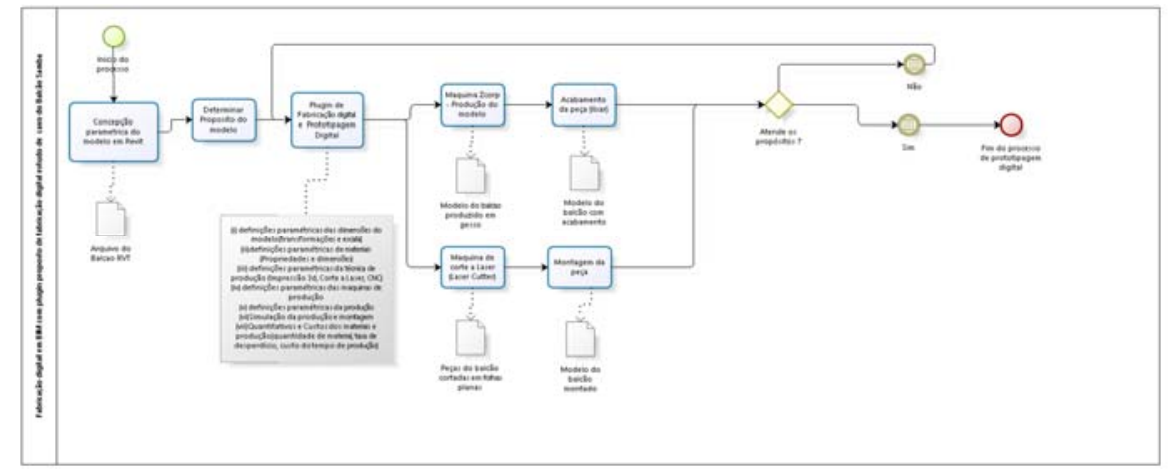

Fonte: os autores

Analisando-se o novo BPMN é possível observar que o fluxo de informação na produção do modelo foi otimizado com o corte de etapas. Com relação ao desafio da interoperabilidade, como o arquivo agrega distintas extensões de exportação para máquinas o fluxo de informações torna-se contínuo. O modelo traz inserido todas as propriedades paramétricas relativas ao protótipo. Toda a preparação da informação pode ser relacionada parametricamente agilizando o processo de fabricação. Em todas as etapas o modelo pode ter alterações parametrizadas. A geometria e as informações só são congeladas (na extensão aceita pela máquina selecionada de acordo com as características do protótipo) na etapa de produção.

\section{CONCLUSÕES}

No mercado atual as ferramentas BIM estão voltadas para a modelagem da informação da construção. Quando se trata de modelos em escala surgem lacunas que precisam ser preenchidas e demandas que necessitam ser supridas. Há um descompasso entre as extensões produzidas pelos softwares e as lidas pelas máquinas. Isso leva ao aumento de etapas e processos que acabam dificultando o fluxo contínuo de informações. Pode-se dizer que o "caminho é tortuoso" na maior parte das vezes. A interoperabilidade fica comprometida e a manipulação do modelo dificultada. 
Surge a necessidade de investimentos em softwares e plug-ins BIM que possam tratar da modelagem da informação dos modelos de forma direcionada ao protótipo. Também as máquinas poderiam oferecer a possibilidade de ler extensões nativas dos softwares BIM. Em contrapartida as empresas que desenvolvem softwares deveriam incluir as informações necessárias à prototipagem digital em suas extensões nativas. Todo o potencial já desenvolvido em BIM deve ser aproveitado nos processos de prototipagem digital.

No modelo BPMN-BIM hipotético (Figura 11) percebe-se que houve uma otimização do processo quando comparado com as situações dos modelos: BPMN-CAD (Figura 6), BPMNBIM (Figura 8), BPMN-BIM otimizado (Figura9). O desafio principal é a inserção das informações paramétricas no processo de produção. Em todos os BPMNs anteriores ao BPMN-BIM hipotético essas informações não estavam aderidas ao protótipo e por isso foi observado poucas mudanças no resultado final do processo de modelagem. Portanto para que haja um salto qualitativo no processo de preparação da informação dos modelos em escala é necessário que as informações paramétricas dos objetos realmente estejam inseridas em todo o processo.

Para que esse cenário venha se tornar realidade é necessária a mobilização de todas as partes interessadas a fim de encontrar um consenso e definir metas e objetivos a médio e a longo prazo. Só assim futuramente será possível estabelecer o grau mais elevado de maturidade BIM.

\section{AGRADECIMENTOS}

Agradecemos ao Programa de Pós-Graduação em Arquitetura, Tecnologia e Cidade da Universidade Estadual de Campinas pelo incentivo ao desenvolvimento deste trabalho e a Capes pelas bolsas de pesquisa destinadas aos alunos.

\section{REFERENNCIAS}

ANDRADE, Max Lira Veras X. de; RUSCHEL, Regina Coeli. INTEROPERABILIDADE DE APLICATIVOS BIM USADOS EM ARQUITETURA POR MEIO DO FORMATO IFC. Gestão \& Tecnologia de Projetos, Brasil, v. 4, n. 2, p.76-111, dez. 2009. ISSN 1981-1543. Disponível em: <http://www.revistas.usp.br/gestaodeprojetos/article/view/50960/55046>. Acesso em: 05 Ago. 2015. doi:http://dx.doi.org/10.4237/gtp.v4i2.102.

BARBOSA N., W. Do projeto à fabricação: um estudo de aplicação da fabricação digital no processo de produção arquitetônica.. 2009, 240f. Dissertação (Mestrado em Arquitetura, Tecnologia e Cidade) - Universidade Estadual de Campinas Faculdade de Engenharia Civil, Arquitetura e Urbanismo, Campinas, 2009.

CELANI, G.; PICCOLI V. The roles of a model. Arquiteturarevista, São Leopoldo, RS, v. 6, n. 1, p. 50-62, jul. 2010

COMPUTER Integrated Construction Research Program. BIM Project Execution Planning Guide. Version 2.0. The Pennsylvania State University, University Park, PA, USA, May 2011. Disponível em <http://bim.psu.edu/default.aspx>. Acesso em: 21 jul.2014.

EASTMAN, C.; TEICHOLZ, P.; SACKS, R.; LISTON, K. Bim Handbook: A Guide to Building Information Modeling for Owners, Managers, Designers, Engineers and Contractors. Hoboken (NJ): John Wiley \& Sons, 2008.

KOLAREVIC, B. Designing and Manufacturing Architecture in the Digital Age. In: ANNUAL CONFERENCE OF EDUCATION AND RESEARCH IN COMPUTER AIDED ARCHITECTURAL DESIGN IN EUROPE,19, 2001, Helsinki. Proceedings ..., Helsinki: Helsinki University of Technology (HUT), p. 117-123, 2001. 
KURZWEIL, R. The singularity is near: When Humans Transcend Biology. Nova lorque. Penguin Books Editora.2005.

PUPO, R. T. Inserção da prototipagem e fabricação digitais no processo de projeto: um novo desafio para o ensino de arquitetura. 2009, 240f. Dissertação (Doutorado em Engenharia Civil) Universidade Estadual de Campinas Faculdade de Engenharia Civil, Arquitetura e Urbanismo, Campinas, 2009.

PUPO, R.; CELANI, G.; DUARTE, J.P. Digital materialization for architecture: definitions and techniques. In: CONGRESSO DA SOCIEDADE IBERO-AMERICANA DE GRÁFICA, 13, 2009, São Paulo. Anais... São Paulo: Faculdade de Arquitetura e Urbanismo da Universidade Presbiteriana Mackenzie, p. 439-442, 2009. 